\title{
Non-canonical function of Bax in stress-induced nuclear protein redistribution
}

\author{
Liora Lindenboim • Elisa Ferrando-May • \\ Christoph Borner · Reuven Stein
}

\begin{abstract}
Bax and Bak (Bax/Bak) are essential pro-apoptotic proteins of the Bcl-2 family that trigger mitochondrial outer membrane permeabilization (MOMP) in a Bcl-2/ Bcl- $\mathrm{x}_{\mathrm{L}}$-inhibitable manner. We recently discovered a new stress-related function for Bax/Bak-regulation of nuclear protein redistribution (NPR) from the nucleus to cytoplasm. This effect was independent of Bax/Bak N-terminus exposure and not inhibited by $\mathrm{Bcl}-\mathrm{x}_{\mathrm{L}}$ over-expression. Here, we studied the molecular mechanism governing this novel noncanonical response. Wild-type (WT) and mutant versions of Bax were re-expressed in Bax/Bak double-knockout mouse
\end{abstract}

L. Lindenboim $\cdot$ R. Stein $(\bowtie)$

Department of Neurobiology, George S. Wise Faculty of Life Sciences, Tel Aviv University, 69978 Ramat Aviv, Israel e-mail: reuvens@post.tau.ac.il

E. Ferrando-May

Department of Biology, Bioimaging Center, University of Konstanz, 78457 Constance, Germany

\section{Borner}

Institute of Molecular Medicine and Cell Research, Albert Ludwigs University Freiburg,

Stefan Meier Strasse 17, 79104 Freiburg, Germany

\section{Borner}

Spemann Graduate School of Biology and Medicine (SGBM), Albert Ludwigs University Freiburg, Albertstrasse 19a, 79104 Freiburg, Germany

\section{Borner}

Excellence Cluster, Centre for Biological Signaling Studies (BIOSS), Albert Ludwigs University Freiburg, Hebelstrasse 25, 79104 Freiburg, Germany embryonic fibroblasts and their ability to promote NPR, apoptotic events, and changes in lamin A mobility was examined. Our results show that, in this system, Bax expression was sufficient to restore NPR such as in WT cells undergoing apoptosis. This activity of Bax was uncoupled from cytochrome $c$ release from the mitochondria (indicative of MOMP) and required its membrane localization, $\alpha$ helices 5/6, and the Bcl-2 homology 3 (BH3) domain. Moreover, enrichment of Bax in the nuclear envelope by the so-called Klarsicht/ANC-1/Syne-1 homology domain effectively triggered NPR as in WT Bax, but without inducing MOMP or cell death. Bax-induced NPR was associated with impairment in lamin A mobility, implying a connection between these two nuclear envelope-associated events. Overall, the results indicate a new MOMP-independent, stress-induced Bax function on the nuclear envelope.

Keywords Apoptosis $\cdot$ Bcl-2 proteins $\cdot$ Nucleus . Nuclear permeability

$\begin{array}{ll}\text { Abbreviations } \\ \text { Bax/Bak } & \text { Bax and Bak } \\ \text { DKO } & \text { Double-knockout } \\ \text { DMEM } & \text { Dulbecco's modified Eagle's medium } \\ \text { FRAP } & \text { Fluorescence recovery after photobleaching } \\ \text { GAPDH } & \text { Glyceraldehyde 3-phosphate dehydrogenase } \\ \text { GFP } & \text { Green fluorescent protein } \\ \text { HA } & \text { Hemagglutinin } \\ \text { KASH } & \text { Klarsicht/ANC-1/Syne-1 homology } \\ \text { MEFs } & \text { Mouse embryonic fibroblasts } \\ \text { MOMP } & \text { Mitochondrial outer membrane } \\ & \text { permeabilization } \\ \text { NE } & \text { Nuclear envelope } \\ \text { NPR } & \text { Nuclear protein redistribution } \\ \text { NPM } & \text { Nucleophosmin }\end{array}$


Q-VD-OPH Quinoline-val-asp(OMe)-CH2-OPH

RFP Red fluorescent protein

WT Wild-type

\section{Introduction}

During apoptosis, proteins translocate from one cellular compartment to another to facilitate the apoptotic process. For example, in response to apoptotic stimuli, the pro-apoptotic Bcl-2 family protein Bax translocates from the cytosol to the mitochondria. There, it undergoes conformational changes which include exposure of its $\mathrm{N}$-terminal region and the formation of homo-oligomers. This leads to mitochondrial outer membrane permeabilization (MOMP), causing additional apoptotic translocation events, with apoptogenic mitochondrial proteins such as cytochrome $c$, Smac/DIA$\mathrm{BLO}$, and HtrA2/Omi being released into the cytosol [1-3]. In the cytosol, cytochrome $c$ promotes apoptosome formation, caspase- 9 activation, and the cleavage and activation of the effector caspases 3 and 7 [4]. Several domains are crucial for Bax-mediated MOMP and cell death. These include the $\mathrm{Bcl}-2$ homology domain $\mathrm{BH} 3$, which participates in homo-oligomerization and the interaction of Bax with the hydrophobic pocket of anti-apoptotic Bcl-2 family proteins, as well as $\alpha$-helices 5 and 6 . The latter are structurally homologous to bacterial toxins and required for the insertion of Bax into the outer mitochondrial membrane and its consequent pore-forming activity.

In addition to the translocation of proteins from the mitochondria to the cytosol, nuclear proteins such as p53, Nur77, Histone H1.2, nucleophosmin (NPM), and nucleolin have been shown to translocate from the nucleus to the cytosol or mitochondria during apoptosis, where they participate in the apoptotic process (for review, see [5]). We recently discovered that stress-induced nuclear protein redistribution (NPR) is regulated by Bax and Bak (Bax/Bak). This Bax/Bakmediated effect is independent of $\mathrm{N}$-terminal conformational changes of Bax or Bak, the apoptosome and caspase activity, and is unaffected by $\mathrm{Bcl}-\mathrm{x}_{\mathrm{L}}$ over-expression [6]. Re-expression of Bax or Bak in Bax/Bak double-knockout (DKO) mouse embryonic fibroblasts (MEFs) restored the stress-induced NPR by a mechanism which resembled that in stressed wild-type (WT) MEFs, since it was not inhibited by the general caspase inhibitor BOC-Asp(OMe)-FMK [6]. The present study explores the molecular details of NPR induced by WT and various mutants of Bax re-expressed in $\mathrm{Bax} / \mathrm{Bak}$ DKO MEFs as the model system. We show that the same domains of Bax are crucial for NPR and for MOMP and cell death. However, targeting Bax to the nuclear envelope (NE) using the Klarsicht/ANC-1/Syne-1 homology (KASH) domain was sufficient for NPR in the absence of MOMP and cell death. Thus, our results point to a novel non-canonical, MOMP-independent action of Bax at the NE which leads to NPR.

\section{Materials and methods}

Materials

All reagents were purchased from Sigma-Aldrich (St. Louis, MO, USA), unless otherwise specified. Quinolineval-asp(OMe)-CH2-OPH (Q-VD-OPH) was purchased from R\&D Systems (Minneapolis, MN, USA).

Cell culture

Primary WT and Bax/Bak DKO MEFs were obtained from Andreas Strasser (The Walter and Eliza Hall Institute of Medical Research, Parkville, Victoria, Australia). They were immortalized by the 3T9 method [7] and grown in high-glucose Dulbecco's modified Eagle's medium (DMEM) supplemented with $10 \%$ heat-inactivated fetal calf serum.

Plasmids

The expression vectors used in this study were: pEGFP (Clontech Laboratories, Mountain View, CA, USA), pEGFPBax (GFP-Bax), $\triangle \mathrm{N}(1-20)$ Bax and FLAG-Bax prepared as described [8]; pcDNA3 HA-Bax (HA-Bax) prepared as described [9]; FLAG-Bcl- $x_{L}$ prepared as described [10]; GFP-Bax L63E [Leu 63 (CTC) was replaced by Glu (GAG) using the forward primer: 5' GAAGCTGAGCGAGTGT GAGAAGCGCATCGGGG and the reverse primer: 5' CCCCGATGCTCTTCTCACACTCGCTCAGCTTC], GFP-Bax $\triangle$ IGDE (deletion of residues 66-69 using the forward primer: 5' GTCTCAAGCGCCTGGACAGTAA CATGGAGC and the reverse primer: 5' GTTACTGTCCA GGCGCTTGAGACACTCGCTC) and GFP-Bax $\Delta \alpha 5 / 6$ (deletion of residues 108-146 using the forward primer: $5^{\prime}$ CCGAGTGGCAGCTGACATGTGGATCCAAGACCAGG GTGGTTGG and the reverse primer: 5' CCAACCACC CTGGTCTTGGATCCACATGTCAGCTGCCACTCGG) were prepared by two-step PCR amplification, using the above-mentioned primers for the first PCR step and GFPBax as a template. For the second step, the first step PCR products were used as templates employing the forward (5' GTCACTCGAGATGGACGGGTCCGGGGAGCAGC) and reverse (5' GTCAGAATTCTTAGCCCATCTTCTTC CAGATGG) primers containing XhoI and EcoRI sites. The final PCR products were cloned into XhoI-EcoRI sites of pEGFP. GFP-Bax 63-65A and GFP-Bax 92-94A were a gift from $\mathrm{Xu}$ Luo (University of Nebraska Medical Center, Omaha, NE, USA); GFP-Mito-Bax was prepared by in-frame insertion of the NheI/AgeI mitochondrial 
targeting fragment from pEYFP-Mito (Clontech Laboratories, Mountain View, CA, USA) into NheI/AgeI sites in GFP-Bax; GFP-Bax S184V was a gift from Richard Youle (National Institutes of Health, Bethesda, MD, USA); Bax Cyt $b 5$ was generated by subcloning a PCR fragment corresponding to the $\mathrm{C}$ terminus of rat microsomal cytochrome b5 (Cyt b5) (aa 100-134, cDNA kindly provided by Stephen Sligar, Urbana, IL, USA) into the PmlI (in Bax) and $X b a I$ (in the multiple cloning site of pcDNA3) sites of $\mathrm{hBax} / \mathrm{pcDNA} 3$, thereby replacing the $\mathrm{C}$ terminus of Bax with that of Cyt $b 5$; HA-Bax NLS was prepared by inserting an $\mathrm{NcoI} / \mathrm{XhoI}$ fragment containing HA-Bax (generated by PCR using pcDNA3 HA-Bax as a template and the forward: 5' GTCACCATGGTCCACCATGGCATACCCATACG and reverse: 5' CGATCTCGAGGCCCATCTTCTTCCAGAT GG primers) into the $N c o I / X h o I$ sites of $\mathrm{pCMV} / \mathrm{myc} /$ nuc (Invitrogen Life Technologies, Paisley, UK). FLAG-Bax KASH was prepared by two-step PCR that joined Bax and KASH sequences in frame. In the first step, the forward: $5^{\prime}$ GTCAGATATCTGACGGGTCCGGGGAGCAGCCCAG AGG and reverse: 5' GGAGGAGCAGCTGCAGGGGTAG GGCGCCCATCTTCTTCCAGATGGTGAGCG primers and pEGFP-Bax as template or the forward: 5' CGCTCACCATCTGGAAGAAGATGGGCGCCCTACCC CTGCAGCTGCTCCTCC and reverse: 5' GTCATCTAGA CTATGTGGGGGGTGGCCCATTGG primers and pTREmBcl-2-KASH [12] as a template were used. For the second PCR step, the products of the first step were used as templates using the forward: 5' GTCAGATATCTG ACGGGTCCGGGGAGCAGCCCAGAGG and the reverse: 5' GTCATCTAGACTATGTGGGGGGTGGCCCATTGG primers and the Bax KASH PCR product was cloned into the EcoRV/XbaI sites of FLAG-pcDNA3 [11];GFP-Bax $\triangle \mathrm{TM}$ and GFP-Bax P168A were prepared as described [8]; pDsRed-Mito was from Clontech Laboratories, CherryLAP2 $\beta$ was a gift from Eran Bacharach (Tel Aviv University, Tel Aviv, Israel), and pHcRed1-Nuc was a gift from Adi Kimchi (Weizmann Institute of Science, Rehovot, Israel); FLAG- $\Delta \alpha 5 / 6$ Bax KASH was prepared by twostep PCR amplification. In the first step, the forward: 5' GTCAGATATCTGACGGGTCCGGGGAGCAGCCCAG AGG and reverse: 5' CCAACCACCCTGGTCTTGGAT CCACATGTCAGCTGCCACTCGG or the forward: $5^{\prime}$ CCGAGTGGCAGCTGACATGTGGATCCAAGACC AGGGTGGTTGG and the reverse: 5' GTCATCTAGACT ATGTGGGGGGTGGCCCATTGG primers and FLAGBax KASH as a template were used. In the second step, the products of the first PCR step were used as templates using the forward: 5' GTCAGATATCTGACGGGTCC GGGGAGCAGCCCAGAGG and reverse: 5' GTCATCTAG ACTATGTGGGGGGTGGCCCATTGG primers. The PCR product of the second step was inserted into the EcoRV/XbaI sites of pcDNA3-FLAG. FLAG- L63E Bax KASH was prepared by two-step PCR amplification. In the first step, the forward: 5' GTCAGATATCTGACGGGTCCGG GGAGCAGCCCAGAGG and reverse: 5' CCCCGATG CTCTTCTCACACTCGCTCAGCTTC or the forward: $5^{\prime}$ GAAGCTGAGCGAGTGTGAGAAGCGCATCGGGG and the reverse: 5' GTCATCTAGACTATGTGGGGGGTGGCCCATTGG primers and FLAG-Bax KASH as a template were used. In the second step, the products of the first PCR step were used as templates using the forward: $5^{\prime}$ GTCAG ATATCTGACGGGTCCGGGGAGCAGCCCAGAGG and reverse: 5' GTCATCTAGACTATGTGGGGGGTGGCCC ATTGG primers. The PCR product of the second step was inserted into the EcoRV/XbaI sites of pcDNA3-FLAG.

Bcl- $x_{\mathrm{L}}$ KASH (with a FLAG tag at its $\mathrm{N}$ terminus) was prepared by two-step PCR that joined $\mathrm{Bcl}-\mathrm{x}_{\mathrm{L}}$ and $\mathrm{KASH}$ in frame. In the first step, the forward: 5' GCTGGAATTCACCATGGATTACAAGGACG and reverse: 5' GGAGG AGCAGCTGCAGGGGTAGGGCTTTCCGACTGAAG AGTGAGCCC primers and FLAG-Bcl- $x_{L}$ as a template or the forward: 5' GGGCTCACTCTTCAGTCGGAAAG CCCTACCCCTGCAGCTGCTCCTCC and reverse: 5' GT CATCTAGACTATGTGGGGGGTGGCCCATTGG primers and pTRE-mBcl-2-KASH as template were used. In the second step, the PCR products of the first step were used as templates using the forward: 5' GCTGGAATTCACC and the reverse: 5' GTCATCTAGACTATGTGGGGGGTGGC CCATTGG primers. The Bcl- $\mathrm{x}_{\mathrm{L}}-\mathrm{KASH}$ PCR product was inserted into the EcoRI/XbaI sites of pcDNA3; Bcl- $\mathrm{x}_{\mathrm{L}} \mathrm{Cyt}$ $b 5$ was generated by subcloning a PCR fragment of rat Cyt $b 5$ (see Bax Cyt $b 5$ above) into the SphI (in Bcl- $\mathrm{x}_{\mathrm{L}}$ ) and $\mathrm{XbaI}$ (in the multiple cloning site of pcDNA3) sites of hBcl$\mathrm{x}_{\mathrm{L}} / \mathrm{pcDNA} 3$, thereby replacing the $\mathrm{C}$ terminus of $\mathrm{Bcl}-\mathrm{x}_{\mathrm{L}}$ with that of Cyt b5; Lamin A-RFP [13] GFP-mini-nesprin2G [14] were a gift from Howard J. Worman (Columbia University, NY, USA); GFP-tBid was a gift from Atan Gross (Weizmann Institute of Science, Rehovot, Israel) and GFP$\mathrm{Bcl}-\mathrm{X}_{\mathrm{S}}$ was prepared as described [9].

\section{Transfection}

Transfection into Bax/Bak DKO MEFs was performed with lipofectamine (Invitrogen Life Technologies, Paisley, UK) according to the manufacturer's instructions. One day before transfection, the cells were seeded at a density of $10^{5}$ cells per well in 12-well plates. When transfections were performed in the presence of Q-VD-OPH, it was added $5 \mathrm{~h}$ after adding the transfection reagents. The ratios of the different DNA vectors were as follows: 1:1 for pEGFP or each of the different Bax-based vectors and pcDNA3; 1:1 for GFP-Bax targeted to different subcellular compartments and pDsRed-Mito, HcRed1-Nuc or Cherry-LAP2B; 1:1:1 for GFP-Bax or FLAG-Bax KASH and Bcl- $\mathrm{x}_{\mathrm{L}}, \mathrm{Bcl}-\mathrm{x}_{\mathrm{L}^{-}}$ KASH or Bcl- $\mathrm{x}_{\mathrm{L}}$ cyt $\mathrm{b}$, and pcDNA3. 
Immunofluorescence staining

Cells were grown in 12 -well plates, $10^{5}$ cells per dish, on 18 -mm cover slips coated with collagen; $24 \mathrm{~h}$ after transfection, cells were fixed and stained with the different antibodies and Hoechst dye 33258, as described previously [15]. The following primary antibodies were used: rabbit anti-nucleolin C23 (H-250) and rabbit anti-calnexin (both from Santa Cruz Biotechnology, Santa Cruz, CA, USA), mouse antiFLAG M5 and rabbit anti-HA (both from Sigma-Aldrich), mouse anti-HA (12CA5, purified from hybridomas), mouse anti-NPM (Zymed Laboratories, San Francisco, CA, USA) mouse anti-histidine TAG (AbD; Serotec, NC, USA), rabbit anti-Bax-N terminal (NT) (Millipore, MA, USA), mouse anti-Bax NT (6A7 antibody) and mouse anti-cytochrome $c$ (both from BD Pharmingen ${ }^{\mathrm{TM}}$, MD, USA) and rabbit antiGAPDH (Cell Signaling Technology, MA, USA). Fluorescent images were captured with a fluorescence microscope (Nikon ECLIPSE TE2000-S) connected to a CCD camera (CoolSNAP HQ; RoPER Scientist) with plan Apo VC $60 \times / 1.4$ oil-immersion objective using Image-Pro PLUS (v.4.5.1) software (Media Cybernetics) and exported to Photoshop (Adobe). To determine the number of cells exhibiting the redistribution effect or apoptotic nuclei (exhibiting nuclear condensation or fragmentation), the fluorescently stained cells were counted under the fluorescence microscope. Confocal fluorescent images were obtained using a Meta Zeiss LSM 510 confocal microscope using Zeiss X63 NA 1.4 objective lens. Images were acquired with Zeiss LSM Image Browser v.4.2.0.121.

\section{FRAP analysis}

Cells were plated on a $35-\mathrm{mm}^{2}$ glass-bottom dish (Greiner Bio-One, Frickenhausen, Germany), transfected with lamin A-RFP with or without additional plasmids and imaged $24 \mathrm{~h}$ later. Imaging medium was similar to growth medium, but consisted of DMEM without phenol red. The imaging setup consisted of an iMIC inverted microscope with an oilimmersion Plan-Apochromatic $60 \times$ objective NA $=1.42$ (Olympus), a Polychrome V system (TILL Photonics, Gräfelfing, Germany), $561 \mathrm{~nm} 75 \mathrm{~mW}$ DPSS laser "Jive" (Cobolt), a polytrope condenser (iMIC Beam-switch for widefield, TIRF, FRAP illumination) and an ANDOR iXon DU 888D EMCCD camera (Andor, Belfast, UK). The microscope was in a $37{ }^{\circ} \mathrm{C}$ temperature-controlled chamber with $5 \% \mathrm{CO}_{2}$ atmosphere. The equipment was controlled by Live Acquisition Software (TILL Photonics). Two baseline images were taken prior to the bleaching. The bleach pulse was for $600 \mathrm{~ms}$ at $80 \%$ laser intensity. Bleaching area was two lines drawn across the nucleus from the nuclear rim through the nucleoplasm. Recovery of fluorescence was monitored at $30-\mathrm{s}$ intervals for $30 \mathrm{~min}$. The average intensity of the fluorescent signal was measured in two regions of interest: rim [a bleached area at the nuclear periphery corresponding to the regions which exhibit higher fluorescent intensity than the nuclear interior (marked by arrow and rectangle, Fig. 7a, right panel)] and nucleoplasm [bleached area in interior of the nucleus (marked by arrowhead, Fig. 7a, right panel)]—using Metamorph software (Molecular Devices, CA, USA). It was then normalized to the change in total fluorescence as $I_{\text {rel }}=T_{0} I_{t} / T_{t} I_{0}$ where $T_{0}$ is total cellular intensity during pre-bleach, $T_{t}$ is total cellular intensity at time $t, I_{0}$ is the average intensity in a region of the pre-bleached nucleus corresponding to the bleach region, and $I_{t}$ is the average intensity in the bleach region at time $t$ [16]. The normalized fluorescence was then plotted against time after bleaching. Mobile fraction (\% recovery) was calculated as $\left[\left(I_{\text {rel }_{t=30}}-I_{\text {rel }_{t=\mathrm{bl}}}\right) \times 100 /\left(1-I_{\text {rel }_{t=\mathrm{bl}}}\right)\right]$, where $I_{\text {rel } t=30}$ is the intensity after $30 \mathrm{~min}$ and $\mathrm{I}_{\mathrm{rel}} t_{t=\mathrm{bl}}$ is the intensity after the bleach.

\section{Results}

Re-expression of Bax in Bax/Bak DKO MEFs restores stress-induced NPR by a mechanism which resembles stress-induced NPR in WT MEFs

In a previous study, we showed that Bax/Bak regulate stressinduced NPR in WT MEFs and that this effect is caspaseindependent and not inhibited by $\mathrm{Bcl}-\mathrm{x}_{\mathrm{L}}$ over-expression [6]. To recapitulate stress-induced NPR, we transiently re-expressed a green fluorescent protein (GFP)-tagged version of Bax in Bax/Bak DKO MEFs [6] and tested whether $\mathrm{Bcl}-\mathrm{x}_{\mathrm{L}}$ is still unable to block this process. Since caspase inhibition does not affect stress-induced NPR [6], this and subsequent experiments were performed in the presence of the broad-spectrum caspase inhibitor, Q-VD-OPH, to attenuate Bax-induced cell death. Note that, in these cells, GFP-Bax transfection alone triggered apoptosis without any additional apoptotic stimuli. As shown in Fig. 1a, GFPBax expression caused redistribution of nucleolin $24 \mathrm{~h}$ after transfection, as indicated by its appearance in the cytoplasm and reduced expression in the nuclei and nucleoli. However, this redistribution was not affected by co-expression with Bcl- $\mathrm{x}_{\mathrm{L}}$, although $\mathrm{Bcl}-\mathrm{x}_{\mathrm{L}}$ effectively inhibited GFP-Baxinduced cytochrome $c$ release, that is, cytochrome $c$ was retained in punctuated mitochondrial structures (Fig. 1a). Similar results were obtained for NPM and histone H1 redistribution (Supplemental Fig. S1) or when hemagglutinin (HA)-Bax was used instead of GFP-Bax (Fig. 1b). Quantification of nucleolin and cytochrome $c$ redistribution by individual cell counting showed that expression of HA-Bax in Bax/Bak DKO MEFs induces nucleolin and cytochrome $c$ redistribution. $\mathrm{Bcl}-\mathrm{x}_{\mathrm{L}}$ did not change the HA-Bax-induced 


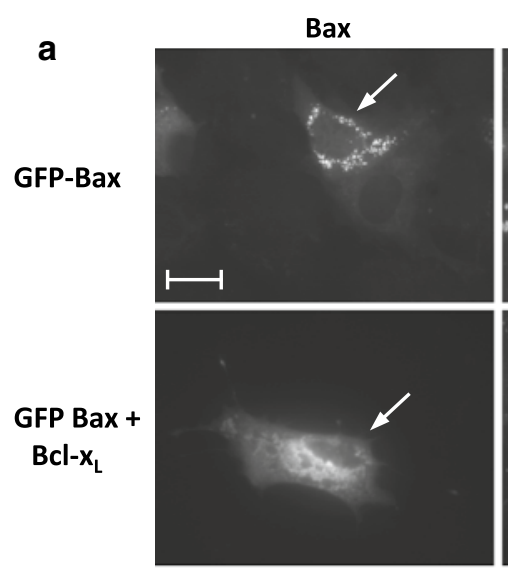

Bax
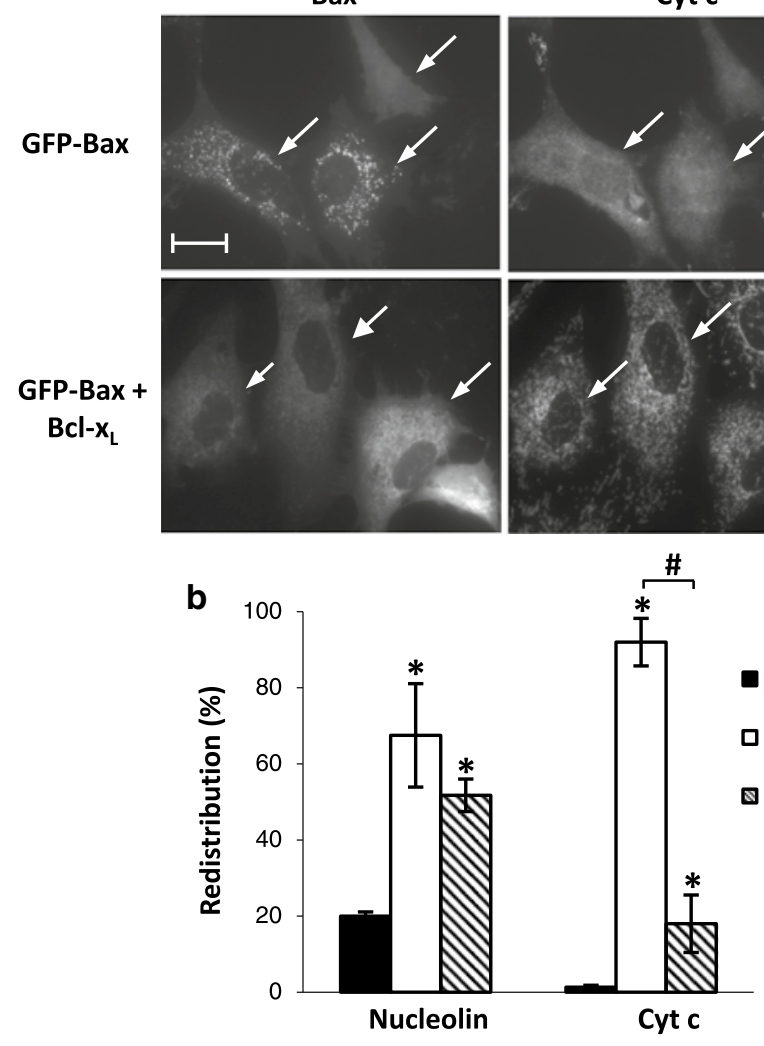

Nucleolin

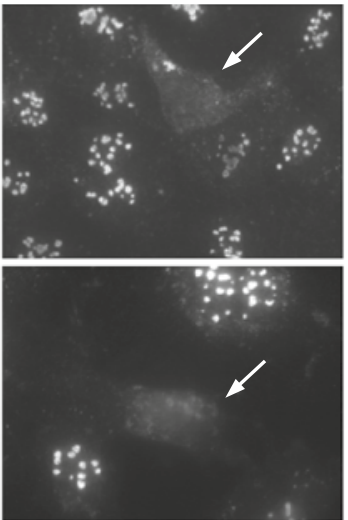

Cyt c
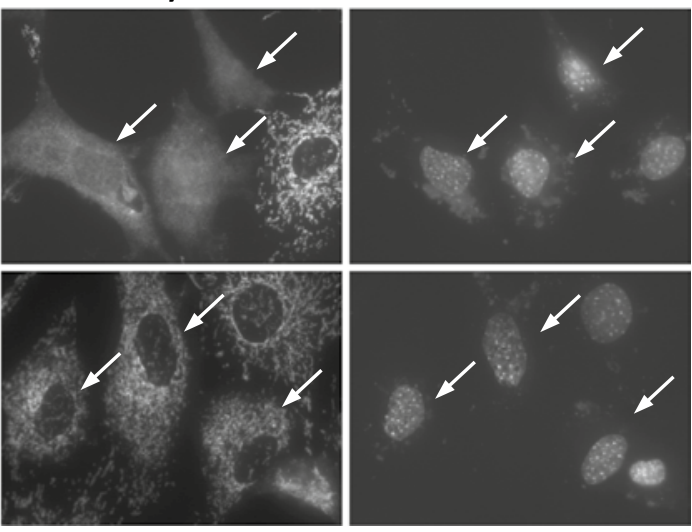

Nuclei

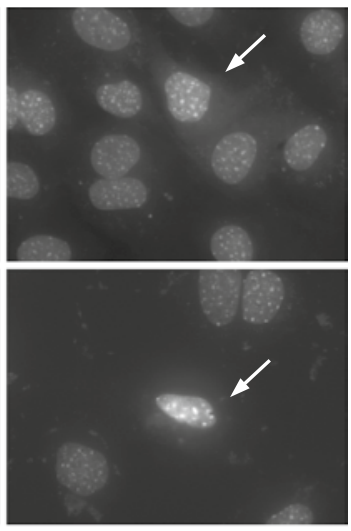

Nuclei

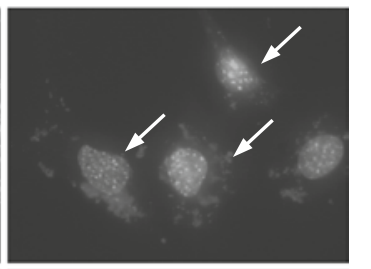

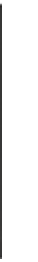

pEGFP

口HA-Bax

هHA-Bax+Bcl-xL

pEGFP-transfected cells, or double-stained with anti-nucleolin or anti-cytochrome $c$ and anti-HA, in HA-Bax-transfected cells. The number of cells exhibiting nucleolin or cytochrome $c$ redistribution was determined microscopically. Presented results are expressed as percentage of cells exhibiting both redistribution and pEGFP or HA-Bax expression, from the total population of pEGFP- or HA-Bax-expressing cells (at least 100 cells). Values are presented as mean $\pm \mathrm{SD}$ (error bars), $(n=3)$. One-way ANOVA revealed a significant effect of treatments $(p<0.001)$ for both nucleolin and cytochrome $c$ redistribution. Tukey's post hoc analysis revealed that the level of both nucleolin and cytochrome $c$ redistribution were significantly higher in the HA-Bax and HA-Bax plus $\mathrm{Bcl}-\mathrm{x}_{\mathrm{L}}$ treatments compared to the control pEGFP treatment $\left({ }^{*} p<0.05\right)$. However, whereas the effect of the HA-Bax plus Bcl- $\mathrm{x}_{\mathrm{L}}$ treatment on cytochrome $c$ release was significantly lower than that of the HA-Bax treatment $\left({ }^{\#} p<0.05\right)$, the effects of these treatments on nucleolin redistribution were similar 
redistribution of nucleolin, but blocked cytochrome $c$ release (Fig. 1b). Moreover, we previously reported that NPR induced by Bax re-expression in Bax/Bak DKO MEFs is also independent of caspases [6]. We therefore found this system suitable for identifying the molecular mechanism governing Bax-induced NPR.

We then examined whether other pro-apoptotic Bcl-2 family members might induce NPR following their expression in Bax/Bak DKO MEFs. The pro-apoptotic proteins tBid (which represents $\mathrm{BH} 3$-only proteins) and $\mathrm{Bcl}-\mathrm{x}_{\mathrm{S}}$ (which acts as a BH3-only protein but also contains a BH4 domain [17]) were expressed in Bax/Bak DKO MEFs. As shown in Fig. 2, neither GFP-tBid nor GFP-Bcl- $x_{S}$ expression induced NPR in these cells. This suggested that the
NPR effect is specifically induced by Bax or Bak, and not by other pro-apoptotic Bcl-2 family proteins.

Bax-induced NPR and MOMP/apoptosis require the same functional domains of Bax

Bax contains nine amphipathic $\alpha$-helices (1-9) [18] and three $\mathrm{BH}$ domains (BH1, BH2, and $\mathrm{BH} 3)$ [19]. The $\mathrm{BH} 3$ domain (containing $\alpha$-helix 2) and $\alpha$-helices 4 and 5 are required for both the oligomerization and killing activity of Bax [20-22]. $\alpha$-Helices 5 and 6 of Bax form a hairpin structure proposed to act as a pore-forming domain, and are necessary for its toxic effect [23]. The $\mathrm{N}^{\prime}$ terminus of Bax, which contains $\alpha$-helix 1 , is important for its conformational
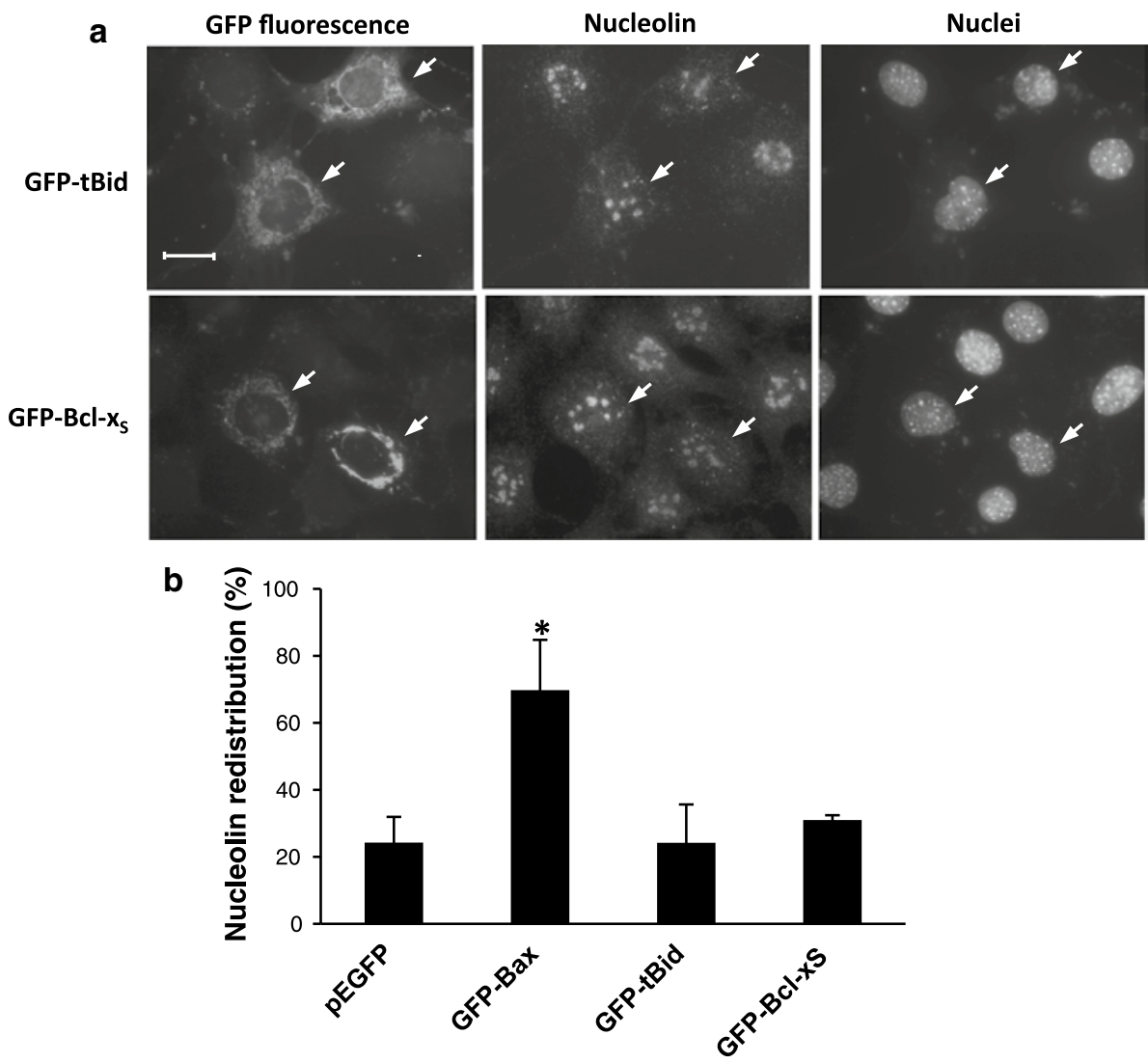

Fig. 2 Expression of GFP-tBid or GFP-Bcl- $\mathrm{x}_{\mathrm{S}}$ in $\mathrm{Bax} / \mathrm{Bak} \mathrm{DKO}$ MEFs does not induce NPR. Bax/Bak DKO MEFs were transiently transfected with pEGFP, GFP-Bax, GFP-tBid or GFP-Bcl-x S $_{\text {. Cells }}$ were stained $24 \mathrm{~h}$ later with anti-nucleolin and Hoechst 33258 dye (to stain nuclei), and visualized by fluorescence microscopy. a The images of each treatment represent the same field visualized separately for detection of GFP fluorescence, nucleolin staining and Hoechst-stained nuclei. Presented results are from a representative experiment (out of at least three independent experiments). Arrows indicate GFP-tBid- or GFP-Bcl- $\mathrm{x}_{\mathrm{S}}$-expressing cells. Bar $20 \mu \mathrm{m}$. b Quantification of the percentage of pEGFP-, GFP-Bax-, GFP-tBidor GFP-Bcl- $\mathrm{x}_{\mathrm{S}}$-expressing cells exhibiting nucleolin redistribution.
Presented results are expressed as the percentage of cells exhibiting both nucleolin redistribution and pEGFP, GFP-Bax, GFP-tBid or GFP-Bcl- $x_{S}$ expression, from the total population of pEGFP-, GFPBax-, GFP-tBid- or GFP-Bcl- $\mathrm{x}_{\mathrm{S}}$-expressing cells (at least 100 cells). Values are presented as mean $\pm \mathrm{SD}$ (error bars) $(n=3)$. A one-way ANOVA revealed a significant effect of treatments $(p<0.001)$. Tukey's post hoc analysis revealed that the effect of the GFP-Bax treatment on nucleolin redistribution was significantly higher than that of the pEGFP control treatment and the GFP-tBid and the GFP-Bcl- $\mathrm{x}_{\mathrm{S}}$ treatments $(* p<0.05)$. Furthermore, no significant difference was observed between the pEGFP control treatment and the GFP-tBid and the GFP-Bcl- $x_{S}$ treatments 

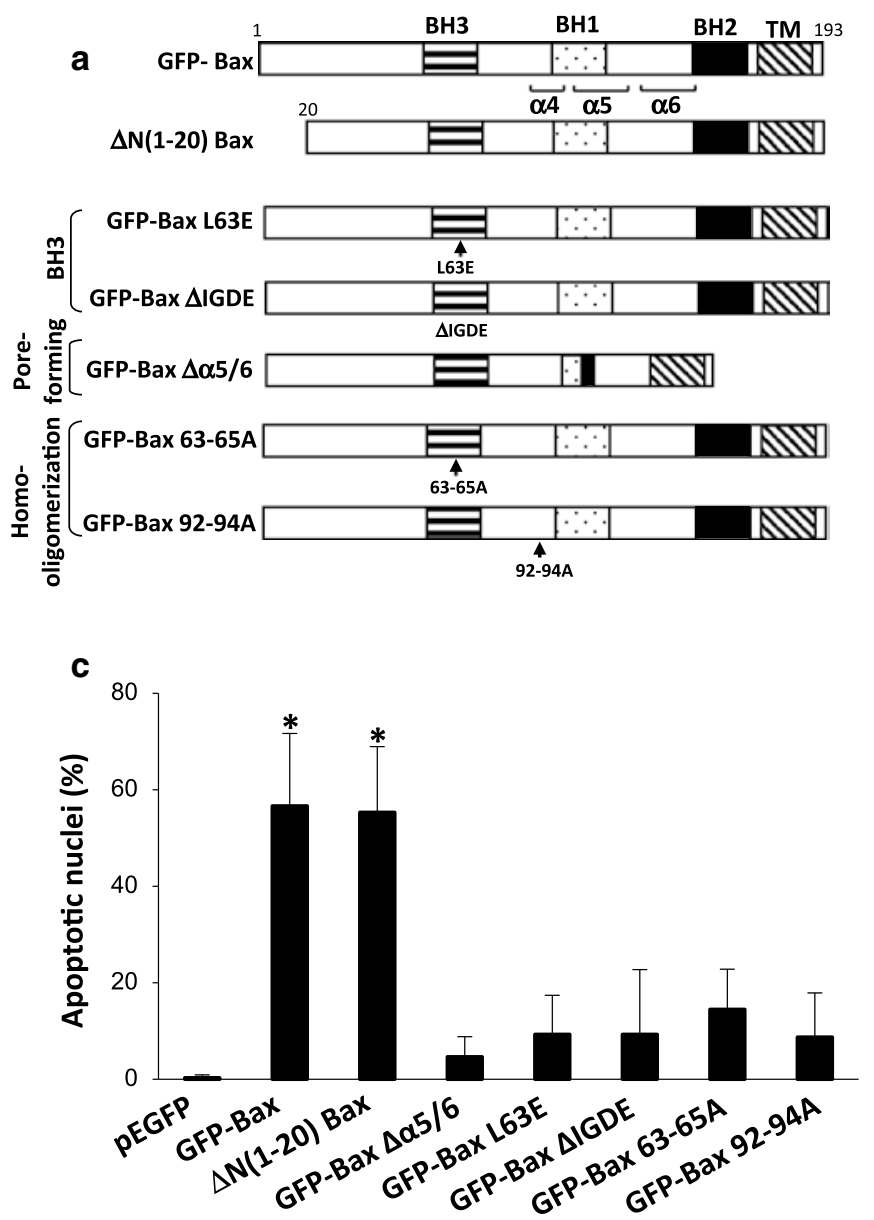

Fig. 3 Identification of Bax domains needed for Bax-induced NPR. a Schematic illustrations of the different Bax constructs used. GFP was fused to the $\mathrm{N}$ terminus. Quantification of the number of cells exhibiting nucleolin redistribution (b), apoptotic nuclei (c) or cytochrome $c$ release (complete; partial) (d) in the transfected cells. Bax/Bak DKO MEFs were transiently transfected with the indicated constructs in the presence of Q-VD-OPH $(20 \mu \mathrm{M})$. Cells were stained $24 \mathrm{~h}$ later with anti-nucleolin or anti-cytochrome $c$ and with Hoechst 33258 dye (to stain the nuclei), and visualized by fluorescence microscopy. The transfected cells were identified by GFP fluorescence, except for $\Delta \mathrm{N}(1-20)$ Bax which has a His-Tag instead of GFP. The latter was double-stained with anti-nucleolin and antihistidine as well as with Hoechst dye. The number of cells exhibiting nucleolin redistribution, cytochrome $c$ release or apoptotic nuclei was determined microscopically. Presented results are expressed as the percentage of cells exhibiting both nucleolin redistribution,

change, mitochondrial targeting and full activation following mitochondrial translocation [24]. To identify which domains are needed for Bax-induced NPR, we generated His-tagged and GFP-tagged Bax mutants in which these various functional domains/helices were mutated or deleted (Fig. 3a). As with the WT Bax above, these mutants were expressed in Bax/Bak DKO MEFs (Supplemental Fig. S2) and tested for their capacity to trigger NPR, cytochrome $c$ release, and cell death. Expression of WT GFP-Bax induced
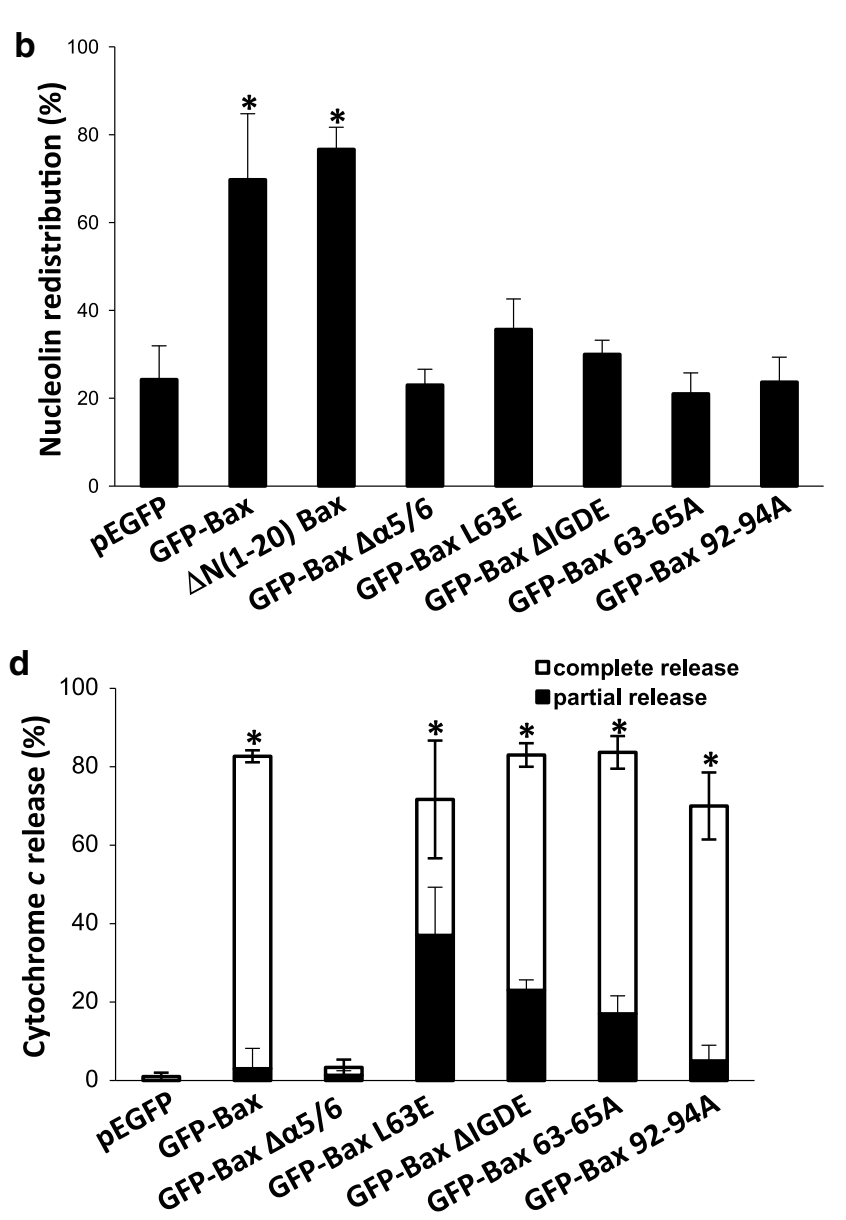

cytochrome $c$ release or apoptotic nuclei and GFP fluorescence or His staining, of the total population of GFP- or His-expressing cells (at least 100 cells). Values are presented as mean \pm SD (error bars) $(n=3)$. A one-way ANOVA revealed a significant effect of treatments for each nucleolin redistribution, apoptotic nuclei and cytochrome $c$ release $(p<0.001)$. Tukey's post hoc analysis revealed that the effect of GFP-Bax and $\Delta \mathrm{N}(1-20)$ Bax treatments on nucleolin redistribution (b) or appearance of apoptotic nuclei (c) was significantly higher than that of the pEGFP control treatment and the rest of the treatments $\left({ }^{*} p<0.05\right)$. In addition, no significant difference was observed between the control pEGFP treatment and all Bax mutants, except $\Delta \mathrm{N}(1-20) \mathrm{Bax}$. The effect of GFP-Bax and all the Bax mutants, except GFP-Bax $\Delta \alpha 5 / 6$ on cytochrome $c$ release (d) was significantly higher than that of the control pEGFP treatment $\left({ }^{*} p<0.05\right)$. No significant difference was observed between the GFP$\operatorname{Bax} \Delta \alpha 5 / 6$ and the control pEGFP treatments

nucleolin redistribution, which was about threefold higher than that induced by the control vector pEGFP (Fig. 3b). This was unaffected by deleting the first 20 amino acids of $\mathrm{Bax}[\Delta \mathrm{N}(1-20)$ Bax]. However, deletions of critical amino acids in the $\mathrm{BH} 3$ domain (L63E and $\triangle \mathrm{IGDE}$ ), in the region essential for homo-oligomerization (63-65A and $92-94 \mathrm{~A})$, and in the pore-forming domain $(\Delta \alpha 5 / 6)$, reduced Bax's ability to promote nucleolin redistribution to about the same level as promoted by pEGFP expression. Scoring 
the cells for apoptotic nuclei yielded the same result as for NPR induction: whereas $\Delta \mathrm{N}(1-20)$ Bax induced condensed nuclei to a similar extent as WT GFP-Bax, the BH3, oligomerization, and pore-forming mutants of GFP-Bax all failed to induce apoptotic nuclei (Fig. 3c). Examination of cytochrome $c$ release in the Bax/Bak DKO MEFs transfected with the different Bax mutants revealed variable degrees of release (Fig. 3d). The toxic WT GFP-Bax caused complete cytochrome $c$ release, as reflected by diffuse staining without any confined punctuated regions. In contrast, the nonapoptotic GFP-Bax mutant $\Delta \alpha 5 / 6$ did not reveal any diffuse cytochrome $c$ staining at all. However, partial cytochrome $c$ release with interspersed punctuated mitochondrial staining was noted for the $\mathrm{BH} 3$ and oligomerization mutants of GFP-Bax (Fig. 3d; Supplemental Fig. S3), although these variants were not cytotoxic (Fig. 3c). The ability of these Bax mutants to partially promote cytochrome $c$ release without triggering NPR indicated that MOMP (cytochrome $c$ release) is insufficient to mediate NPR. Taken together, our results suggested that Bax-induced NPR requires the same domains as Bax-induced cell death and that NPR and cytochrome $c$ release are uncoupled.

Targeting of Bax to the NE triggers NPR, but not MOMP/ apoptosis

We hypothesized that the non-canonical action of Bax on NPR might be exerted at a particular intracellular site such as the NE. To test this, we targeted Bax to specific subcellular localizations using the following constructs (Fig. 4a): GFP-Mito-Bax, (targets Bax to the mitochondria using the cytochrome $c$ oxidase targeting sequence [25]), GFP-Bax S184V (targets Bax to the mitochondria due to the $\mathrm{S} 184 \mathrm{~V}$ mutation which results in constitutive mitochondrial localization of Bax [26]), Bax Cyt b5 (targets Bax to the ER using the cytochrome b5 targeting sequence [27]), HA-Bax NLS (targets Bax to the nucleoplasm due to the addition of nuclear localization sequences [28]), FLAG-Bax KASH (targets Bax to the $\mathrm{NE}$ via the KASH domain, a sequence responsible for targeting nesprin-2 to the outer NE [29]), GFP- $\Delta \mathrm{TM}$ Bax (targets Bax to the cytosol due to deletion of the Bax transmembrane domain), and GFP-Bax P168A (targets Bax to the cytosol due to the P168A mutation, which prevents translocation of Bax to mitochondria [8]). Following transfection into Bax/Bak DKO MEFs, we tested the correct subcellular localization of the different Bax constructs by co-immunostaining with organelle-specific antibodies or expression of fluorescent organellespecific marker proteins. As expected, GFP-Bax $\Delta \mathrm{TM}$ was observed throughout the cell like glyceraldehyde 3-phosphate dehydrogenase (GAPDH), GFP-Bax P168A exhibited a diffuse cytoplasmic distribution, Bax Cyt $b 5$
Fig. 4 The effect of Bax targeting to different subcellular compartments on NPR, cell death and cytochrome $c$ release. a Schematic illustrations of the different Bax constructs used to target Bax to mitochondria, ER, nucleus or cytosol. Quantification of the number of cells exhibiting nucleolin redistribution (b), apoptotic nuclei (c) or cytochrome $c$ release $(\mathbf{d})$ in the transfected cells. Bax/Bak DKO MEFs were transiently transfected with the indicated constructs in the presence of Q-VD-OPH $(20 \mu \mathrm{M})$. Cells were stained $24 \mathrm{~h}$ later with anti-nucleolin or anti-cytochrome $c$ and with Hoechst 33258 dye (to stain the nuclei), and visualized by fluorescence microscopy. To visualize Bax constructs which do not contain GFP, cells were double-stained with anti-nucleolin or anti-cytochrome $c$ and with anti-Bax NT (for Bax Cyt b5) or anti-HA (for HA-Bax NLS) or anti-FLAG (for FLAG-Bax KASH) antibodies and with Hoechst dye. Thereafter, the number of cells exhibiting nucleolin redistribution, cytochrome $c$ release or apoptotic nuclei was determined microscopically as described in Fig. 3. Values are presented as mean \pm SD (error bars) $(n=3)$. A one-way ANOVA revealed a significant effect of treatments for each nucleolin redistribution, apoptotic nuclei and cytochrome $c$ release $(p<0.001)$. Tukey's post hoc analysis revealed that the effects of GFP-Bax and the Bax variants GFP-Mito-Bax, GFP-Bax S184V, Bax Cty b5 and FLAG-Bax KASH on nucleolin redistribution (b) were significantly higher than the similar effects of the control pEGFP treatment and the Bax variants HA-Bax NLS, GFP-Bax $\Delta \mathrm{TM}$ and GFP-Bax P168A $(* p<0.05)$; the effects of GFP-Bax and the Bax variants GFP-Mito-Bax, GFP-Bax S184V and Bax Cty b5 on apoptotic nuclei (c) or cytochrome $c$ release (d) were significantly higher than the similar effects of the control pEGFP treatment and the Bax variants HA-Bax NLS, FLAG-Bax KASH, GFP-Bax $\Delta$ TM and GFP-Bax P168A $(* p<0.05)$

exhibited similar tubular distribution as the ER marker calnexin, GFP-Mito Bax and GFP-Bax S184V exhibited similar punctuated distribution as the mitochondrial marker pDsRed-Mito, HA-Bax NLS exhibited similar nucleoplasm distribution as the nuclear marker HcRed1Nuc, and FLAG-Bax KASH exhibited similar NE distribution as the NE protein Cherry-LAP2 $\beta$ (Supplemental Fig. S4), although it was also observed in other regions, e.g., the ER. We then tested the ability of the variously targeted Bax constructs to induce NPR, cytochrome $c$ release, and cell death. As shown in Fig. 4b, targeting Bax to the mitochondria, the ER, or the NE effectively promoted nucleolin redistribution. This was not the case with the cytoplasmic and nucleoplasmic constructs, which also did not induce cytochrome $c$ release or apoptosis, whereas mitochondria- and ER-targeted Bax provoked these two responses, as expected (Fig. 4c, d). The most interesting Bax variant was FLAG-Bax KASH. This variant, which was associated with the NE, did not provoke either cytochrome $c$ release or apoptosis (Figs. 4c, $\mathrm{d}, 5)$, but induced nucleolin redistribution which was as effective as that induced by WT Bax (Figs. 4b, 5). This latter observation reinforced the notion that Bax-induced NPR differs from its canonical, pro-apoptotic function in the mitochondria, ER or both, and that this novel function of Bax might be performed at the NE, the site where NPR occurs. 

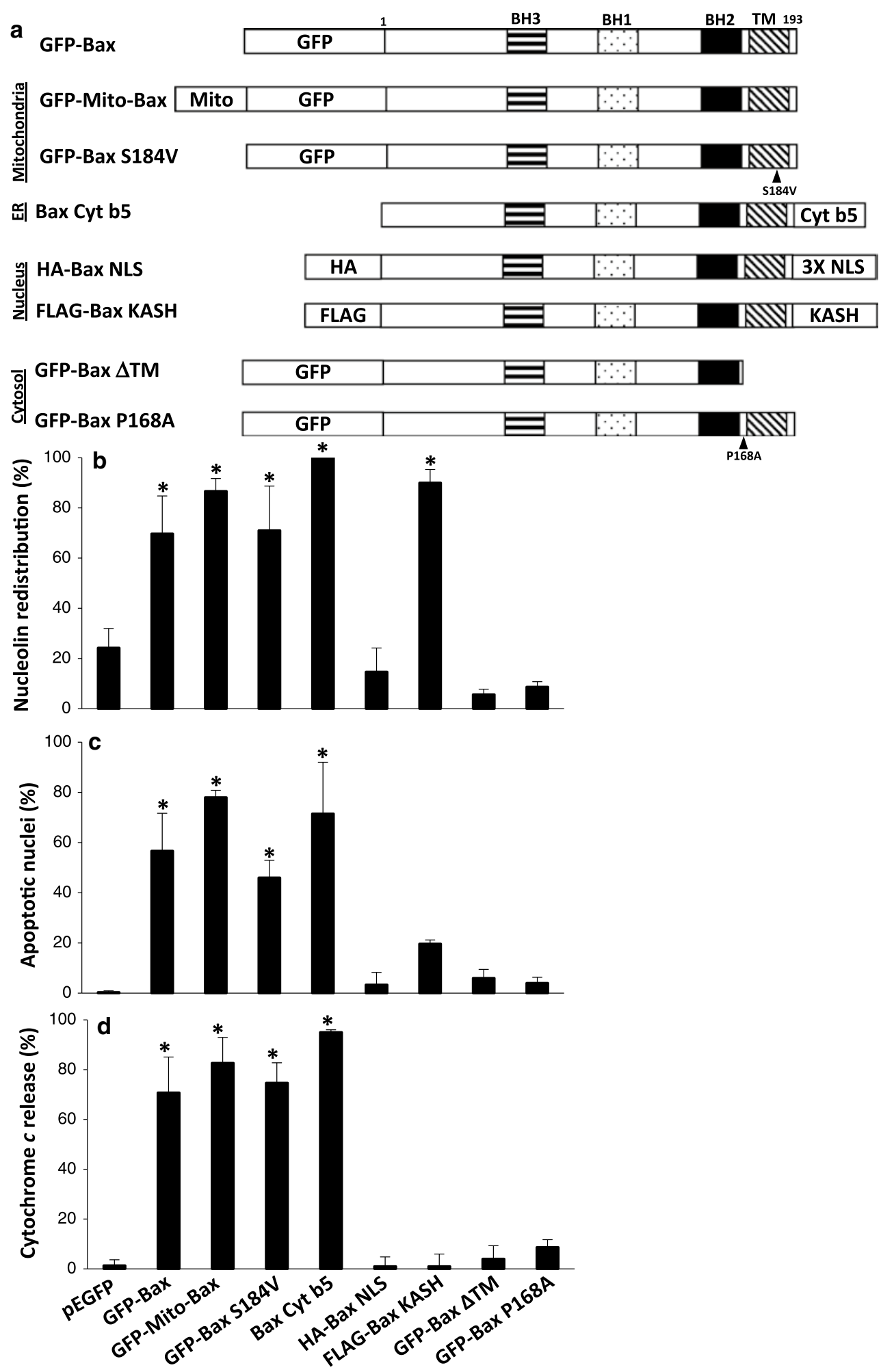

FLAG-Bax KASH-induced NPR is also caspaseindependent and not inhibited by $\mathrm{Bcl}-\mathrm{x}_{\mathrm{L}}$

Because Bax KASH uncouples MOMP and cell death from NPR, this Bax version serves as an ideal model for studying Bax-induced NPR without interfering with its pro-apoptotic function. Therefore, we further characterized the NPR effect induced by expressing FLAG-Bax KASH in Bax/Bak DKO MEFs and compared it to that of a control KASH containing the GFP-mini-nesprin-2G construct. 

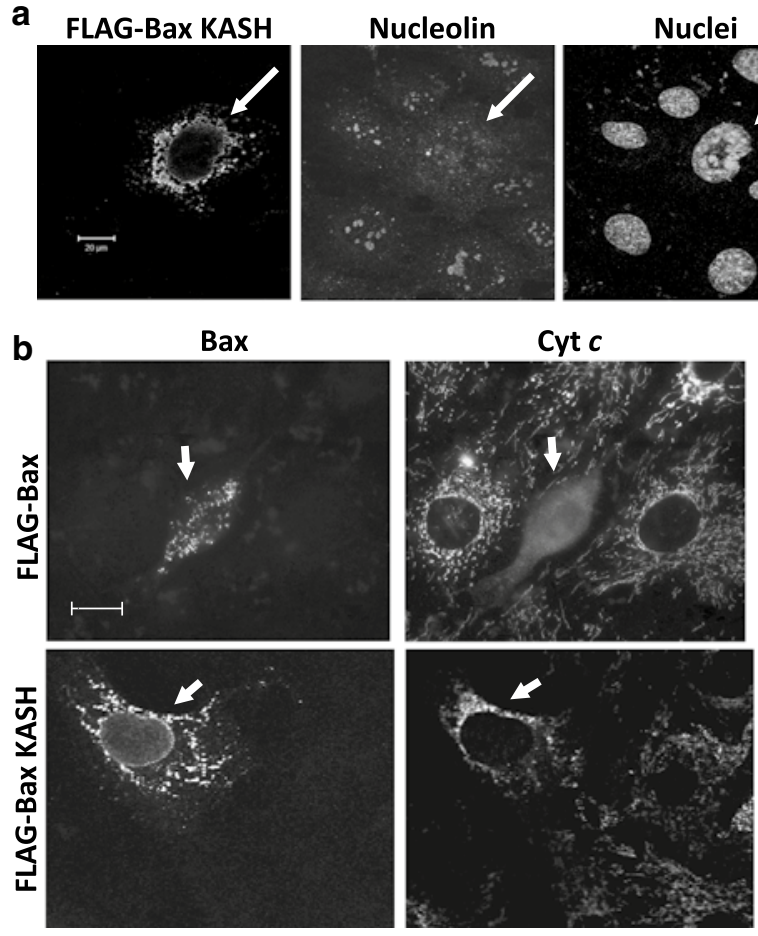

Fig. 5 FLAG-Bax KASH is associated with the NE and does not induce apoptotic effects [MOMP (cytochrome $c$ release) and nuclear condensation]. Bax/Bak DKO MEFs were transiently transfected with FLAG-Bax KASH and 24 h later were stained with anti-FLAG, anti-nucleolin, and Hoechst 33258 dye (to detect the nuclei) (a) or double-stained with anti-Bax NT and anti-cytochrome $c(\mathbf{b})$. Thereafter, the cells were visualized by confocal fluorescence microscopy (a) or fluorescence microscopy (b). The images of each type of staining represent the same field visualized separately for the detection of antibody staining and Hoechst-stained nuclei (a) or Bax NT (Bax) and cytochrome $c$ (Cyt $c$ ) staining. The results are from a representative experiment (out of at least three independent experiments). Bar $20 \mu \mathrm{m}$

As shown in Fig. 6a, FLAG-Bax KASH induced redistribution of all three nuclear proteins-nucleolin, NPM, and histone H1-which was 2-3 fold higher than that caused by GFP-mini-nesprin-2G, indicating that the NPR effect is due to Bax and not the KASH domain alone (Fig. 6b). The FLAG-Bax KASH-induced redistribution of the different nuclear proteins exhibited similar patterns as previously described for stress-induced NPR [6]. Accordingly, NPM was evenly distributed in the cytoplasm. Nucleolin exhibited a granular cytoplasmic staining pattern and reduced expression in nuclei, and the nuclear staining of H1 was markedly reduced. The ability of FLAG-Bax KASH to promote redistribution of a previously expressed NLS-containing reporter was also examined. Bax/Bak DKO MEFs were transfected with the pHcRed1-Nuc vector, which directs nuclear targeting of the NLS-containing reporter HcRed1. After $24 \mathrm{~h}$, these cells were re-transfected with either FLAG-Bax $\mathrm{KASH}$ or GFP-mini-nesprin-2G. After an additional $24 \mathrm{~h}$, the percentage of reporter-expressing cells that exhibited cytosolic HcRed1 was determined microscopically. As exemplified by the representative cells depicted in supplemental Fig. S5 and confirmed by quantitative analysis, similar to its effect on endogenous nuclear proteins, FLAG-Bax KASH was substantially and significantly more effective in promoting redistribution of the nuclear reporter compared to GFP-mini-nesprin-2G (78 \pm 5.2 vs. $18.6 \pm 2.3 \%$, respectively; $p=0.000658$ two-tailed $t$ test, $n=3$ ). Importantly, as seen for WT Bax, co-expression of Bcl- $\mathrm{x}_{\mathrm{L}}$ with FLAGBax KASH or treatment of FLAG-Bax KASH transfectants with the caspase inhibitor Q-VD-OPH did not impede the ability of this construct to induce NPR (Fig. 6c), confirming that this process is non-canonical, that is, independent of caspases and not inhibited by $\mathrm{Bcl}-\mathrm{x}_{\mathrm{L}}$. Moreover, mutation of the $\mathrm{BH} 3$ domain (L63E) or deletion of $\alpha$-helices 5/6 in FLAG-Bax KASH substantially inhibited the NPR effect (Fig. 6d), but not targeting to the NE (although it did increase its ER-like localization) (Supplemental Fig. S6). These results indicated that FLAG-Bax KASH-induced NPR recapitulates the NPR induced by WT Bax.

The inability of Bcl- $\mathrm{x}_{\mathrm{L}}$ to inhibit FLAG-Bax KASHinduced NPR might have been due to a different subcellular localization of the two proteins. To test this, we generated Bcl- $\mathrm{x}_{\mathrm{L}} \mathrm{KASH}$ and Bcl- $\mathrm{x}_{\mathrm{L}}$ Cyt $b 5$ to target $\mathrm{Bcl}-\mathrm{x}_{\mathrm{L}}$ to the NE or ER, respectively. However, as with WT Bcl- $\mathrm{x}_{\mathrm{L}}$, neither of the targeted $\mathrm{Bcl}-\mathrm{x}_{\mathrm{L}}$ constructs was capable of impairing nucleolin redistribution triggered by FLAG-Bax KASH (Fig. 6e). These results demonstrated that the inability of Bcl- $\mathrm{x}_{\mathrm{L}}$ to inhibit the FLAG-Bax KASH-induced NPR effect is not due to different subcellular localization of the two proteins.

Expression of Bax or Bax KASH impairs lamin A mobility

It is reasonable to assume that Bax regulates NPR by changing NE permeability. Therefore, it is interesting to examine if such changes are associated with alterations of the lamina, since an intact lamina is one prerequisite for a functional NE permeability barrier. To gain some insight into such functionality, we studied the effect of Bax on mobility of lamin A, a major constituent of lamina [30], to detect potential alterations. Lamin A mobility was analyzed using fluorescence recovery after photobleaching (FRAP), an assay which assesses the dynamics of fluorescent proteins in living cells. Bax/Bak DKO MEFs were transfected with lamin A-RFP alone or together with FLAG-Bax, in the presence or absence of the general caspase inhibitor Q-VD-OPH or with Bcl- $\mathrm{x}_{\mathrm{L}}$ or FLAG-Bax KASH. After $24 \mathrm{~h}$, a region at the nuclear lamina was bleached and the recovery of fluorescent lamin A-RFP was monitored at both the nuclear rim and the nucleoplasm over a period of up to $30 \mathrm{~min}$ (Fig. 7a, b). As previously shown in other cell types (for review see [30] 

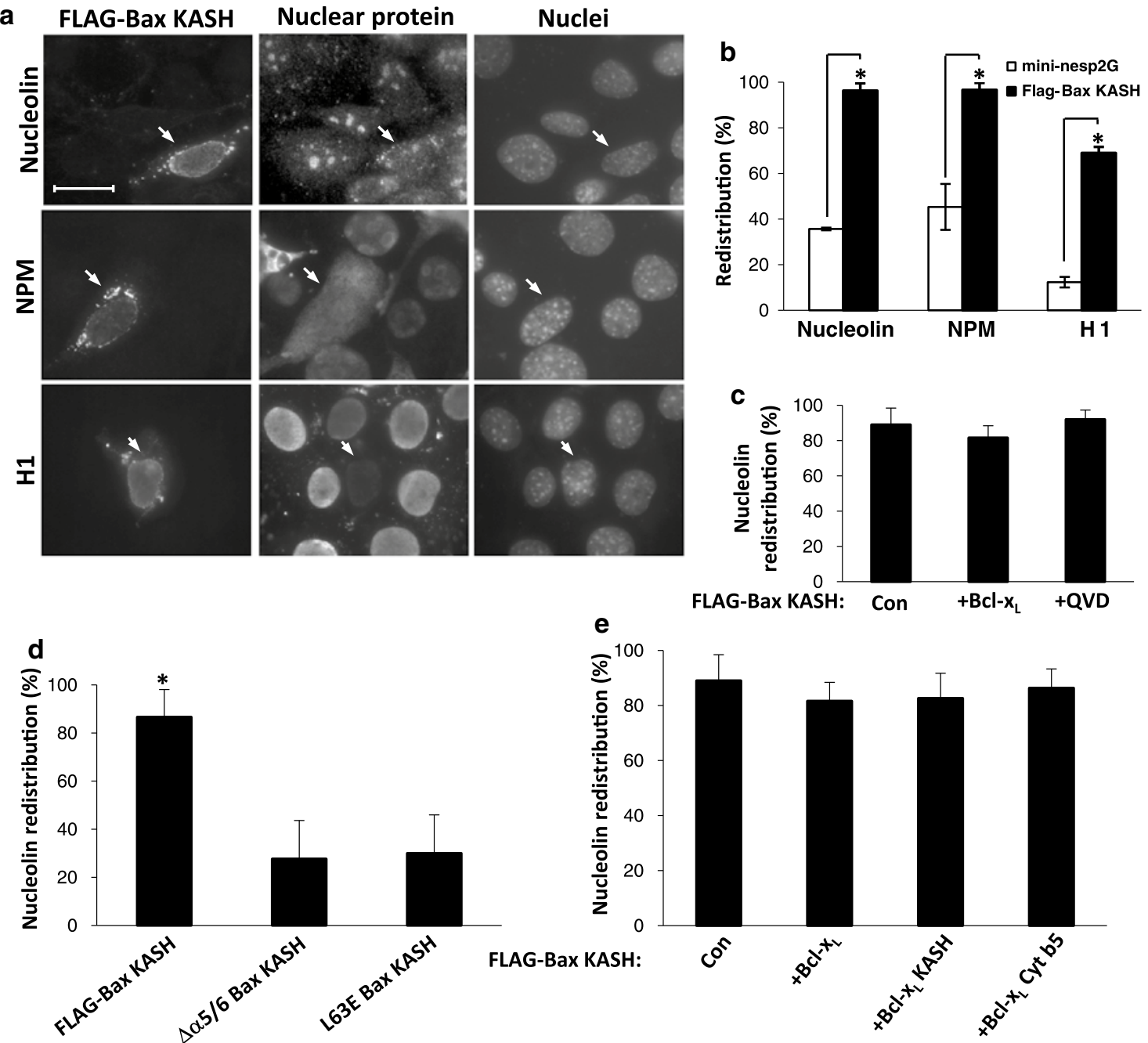

e

FLAG-Bax KASH: Con +Bcl- $\mathrm{x}_{\mathrm{L}} \quad+\mathrm{QVD}$

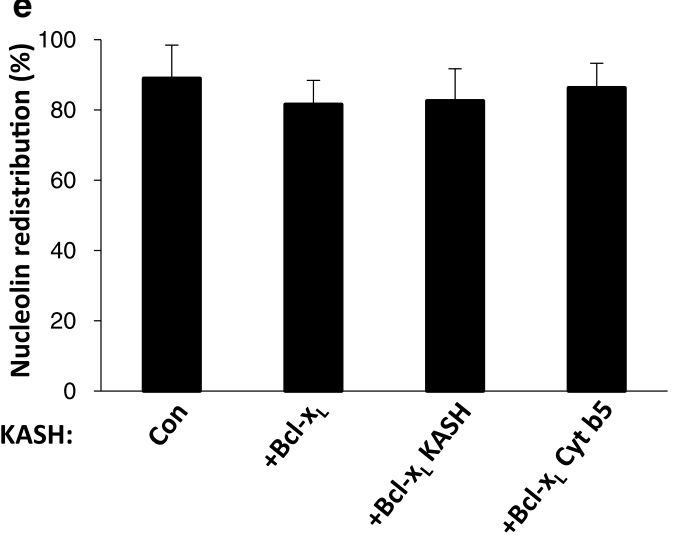

Fig. 6 Characterization of FLAG-Bax KASH-induced NPR. Bax/Bak DKO MEFs were transiently transfected with: FLAG-Bax KASH, FLAG-Bax KASH harboring the $\Delta \alpha 5 / 6$ or L63E mutation, or GFP-mini-nesprin-2G, or co-transfected with FLAG-Bax KASH and each of the indicated Bcl- $\mathrm{x}_{\mathrm{L}}$ constructs. Cells were stained $24 \mathrm{~h}$ later for the indicated nuclear proteins and FLAG-Bax KASH (anti-FLAG or anti-Bax NT) and visualized by fluorescence microscopy. a Representative images of nucleolin, NPM and histone H1 (H1) staining of FLAG-Bax KASH transfected cells. Images of each nuclear protein staining represent the same field visualized separately for detection of FLAG (FLAG-Bax KASH), nuclear protein or Hoechst (nuclei) staining. Presented results are from a representative experiment (out of at least four independent experiments). Arrows indicate FLAGBax KASH -expressing cells. Bar $20 \mu \mathrm{m}$. b Quantification of the percentage of cells exhibiting nucleolin, NPM and histone H1 (H1) redistribution in FLAG-Bax KASH- or GFP-mini-nesprin-2G (mininesp2G) expressing cells. GFP-mini-nesprin-2G-expressing cells were identified by GFP fluorescence whereas FLAG-Bax KASHexpressing cells were identified by immunofluorescence staining. Values are presented as mean $\pm \mathrm{SD}$ (error bars) $(n=3)$. ${ }^{*} p<0.01$

and references therein), in Bax/Bak DKO MEFs transfected with lamin A-RFP alone, the influx of fluorescent lamin A protein into the bleached area was inefficient at both the compared to GFP-mini-nesprin-2G, two-tailed $t$ test. c Quantification of the percentage of cells exhibiting nucleolin redistribution in FLAG-Bax KASH-expressing cells without, (Con) or with, Bcl- $\mathrm{x}_{\mathrm{L}}$, or $20 \mu \mathrm{M}$ Q-VD-OPH (QVD). Values are presented as mean $\pm \mathrm{SD}$ (error bars) $(n=3)$. A one-way ANOVA did not reveal a significant effect of treatments. d Quantification of the percentage of cells exhibiting nucleolin redistribution in FLAG-Bax KASH or FLAGBax KASH mutants as indicated. Values are presented as mean \pm SD (error bars) $(n=3)$. A one-way ANOVA revealed a significant effect of treatment $(p<0.001)$. Tukey's post hoc analysis revealed that the effect of FLAG-Bax KASH on nucleolin redistribution was significantly higher than in the two FLAG-Bax KASH mutants $(* p<0.05)$. e Quantification of the percentage of cells exhibiting nucleolin redistribution in FLAG-Bax KASH-expressing cells without (Con) or with Bcl- $\mathrm{x}_{\mathrm{L}}, \mathrm{Bcl}-\mathrm{x}_{\mathrm{L}} \mathrm{KASH}$ or Bcl- $\mathrm{x}_{\mathrm{L}}$ Cyt $b 5$. Bax-expressing cells were identified by staining with anti-Bax NT antibodies. The percentage of the different FLAG-Bax KASH-expressing cells exhibiting nucleolin redistribution was determined as described in Fig. 3. Values are presented as mean $\pm \mathrm{SD}$ (error bars) $(n=3)$. A one-way ANOVA did not reveal a significant effect of treatment

nuclear rim and in the nucleoplasm (Fig. 7a, b). Quantitative analysis (Fig. 7c) revealed a low-mobile fraction $(19.7 \pm 3$ and $17.9 \pm 2.8 \%$ at these two sites, respectively) of lamin 

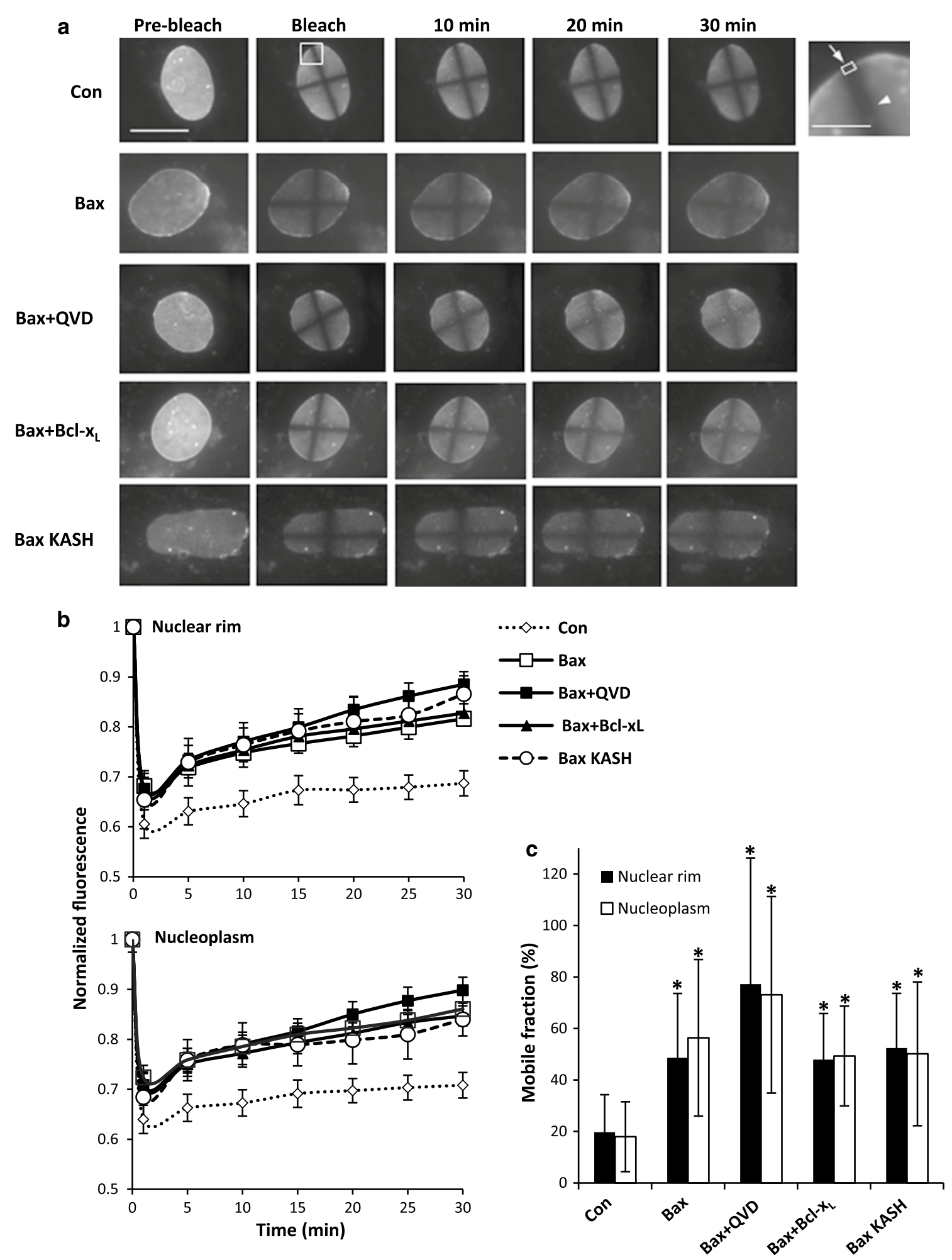

A-RFP, 30 min after photobleaching ( $n=23$ cells). In contrast, co-expression with Bax or Bax KASH increase lamin A-RFP mobility (Fig. 7a and b) resulting in a significant increase in its mobile fraction $(48.6 \pm 4.7$ and $56.3 \pm 5.7 \%$ for Bax; $52.4 \pm 6.4$ and $50.1 \pm 8.4 \%$ for Bax KASH, at

the nuclear rim and nucleoplasm, respectively) ( $n=25$ and 11 cells respectively). Notably, a significant increase in the mobile fraction of lamin A-RFP was also induced when the cells were co-transfected with FLAG-Bax and Bcl- $\mathrm{X}_{\mathrm{L}}(47.9 \pm 3.7$ and $49.3 \pm 4 \%$ at the nuclear rim and 
Fig. 7 Expression of Bax or Bax KASH increases lamin A mobility in the nuclear rim and nucleoplasm. RFP-lamin A was transiently transfected into Bax/Bak DKO MEFs without (Con) or with FLAGBax (Bax) [untreated or treated with $20 \mu \mathrm{M}$ Q-VD-OPH $(Q V D)$ ] or FLAG-Bax and FLAG-Bcl-x $\mathrm{L}_{\mathrm{L}}\left(\mathrm{Bcl}-\mathrm{x}_{\mathrm{L}}\right)$ or FLAG-Bax KASH (Bax $K A S H) ; 24 \mathrm{~h}$ after transfection, live cells expressing lamin A-RFP were visualized microscopically and showed nucleoplasmic and nuclear rim fluorescence (Pre-bleach). Regions at the nuclear rim and nucleoplasm were bleached and fluorescence recovery within these bleached regions was then followed over a period of up to $30 \mathrm{~min}$. A representative region is marked by a square in the bleach image of the top panel. Magnification $(\times 5)$ of this region is shown to the right of the top panel. The arrow and rectangle indicate the region representing the rim, and the arrowhead the region representing the nucleoplasm. Bar $4 \mu \mathrm{m}$. a Representative images for each of the treatments. Bar $20 \mu \mathrm{m}$. b Quantitative analysis of fluorescence in the region of interest in the nuclear rim and nucleoplasm. Presented values are of normalized fluorescence after photobleaching in the nuclear rim (upper panel) and nucleoplasm (lower panel), expressed as mean \pm SE (error bars; $n=11-24$ cells). c The mobile fraction (\% recovery as described in "Materials and methods") values for each curve are shown. Values are presented as mean \pm SD (error bars) $(n=3)$. ANOVA with repeated measures of lamin A mobile fractions in the nuclear rim versus nucleoplasm in the five treatments reveled a significant effect only for the treatments $(p<0.001)$. Tukey's post hoc analysis revealed that the level of lamin A mobile fraction was similar and significantly higher in all treatments compared to the control treatment $(* p<0.05)$

nucleoplasm, respectively) ( $n=24)$ or treated with Q-VD$\mathrm{OPH}(77.2 \pm 10.2$ and $73 \pm 7.9 \%$, respectively) $(n=23)$ (Fig. 7c). In fact, the latter was even higher than with Bax alone. Taken together, our results indicated that both Bax and Bax KASH impair the integrity of the lamin A network via a mechanism that is unaffected by $\mathrm{Bcl}-\mathrm{x}_{\mathrm{L}}$ over-expression or caspase inhibition, suggesting that Bax affects NPR via its effect on lamin A network.

\section{Discussion}

Bax and Bak are essential mediators of apoptotic stimuli. Their pro-apoptotic action encompasses many aspects, such as direct or indirect perforation of the mitochondrial outer membrane [21], regulation of mitochondrial fission [31], and promotion of $\mathrm{Ca}^{2+}$ leakage through the ER membrane [32]. All these processes are associated with a conformational change at the N' termini of Bax and Bak, and they are effectively inhibited by Bcl-2-like pro-survival proteins. Recently, we showed that Bax and Bak can also regulate stress-induced NPR. This function seems to be mediated via a non-canonical, novel mechanism that is uncoupled from $\mathrm{Bcl}-\mathrm{x}_{\mathrm{L}}$ inhibition and N-terminal exposure [6]. Here, we substantiated these results and explored the molecular details of this new process by directly studying the effect of over-expressed Bax on NPR in Bax/Bak DKO MEFs. Our results show that Bax requires the same functional domains for its NPR effect as for promoting cell death (BH3, oligomerization, and pore-forming domains). However, as previously shown, Bax-induced NPR is not blocked by Bcl- $\mathrm{x}_{\mathrm{L}}$ over-expression or caspase inhibition. Strikingly, the Bax KASH variant which exhibited enriched expression in the NE provoked NPR in a similar fashion as WT Bax or Bax targeted to mitochondria or the ER, but it no longer induced apoptosis. With this form of Bax, we can therefore study the impact of Bax on NPR independently of its effect on apoptosis. We also found that Bax-induced NPR is associated with impairment in the integrity of the laminar meshwork and this effect was not inhibited by $\mathrm{Bcl}-\mathrm{x}_{\mathrm{L}}$ over-expression or caspase inhibition. Thus, our findings here lend support to a novel function of Bax on the NE that occurs in parallel to apoptosis induction but is uncoupled from its canonical action on MOMP.

What might be the mechanism by which Bax promotes NPR? The targeting experiments suggest that at least part of the effect is mediated by a Bax localized to the NE. Although the current view is that mitochondria and the ER are the major sites of action of Bax/Bak, a nuclear function for these proteins has also been proposed. Bax has been reported to be localized in nuclei of healthy mammalian cells [33-35]. Moreover, during apoptosis, in addition to translocation to the mitochondria and ER, Bax can also translocate to the NE [36-38]. Thus, we postulate that, in response to apoptotic stimuli, Bax not only causes permeabilization of the outer mitochondrial (MOMP) and ER membranes [39] but a certain NE-associated proportion of it may also be capable of perforating the NE. The mechanism underlying this NE permeabilization is currently unknown. However, it is unlikely to involve the nuclear export system or the degradation of nuclear pore components, because the nuclear export inhibitor leptomycin B, calpain inhibitor (data not shown), and caspase inhibitors did not impede stress-induced NPR, and stress- or Bax-induced NPR was not affected by the treatment with pan-caspase inhibitors. Rather, we propose that Bax somehow destabilizes the nuclear membrane and therefore leads to a rearrangement of the nuclear lamina and its associated proteins. Interestingly, some lamin A mutations which cause laminopathy have been shown to increase lamin A mobility [40]. Alternatively, Bax may interact with one or more nucleoskeleton proteins and linker of nucleoskeleton- and cytoskeleton-complex proteins, such as nesprins and SUN proteins, thereby perturbing the nuclear lamina. Another possibility is that Bax proteins form pores in the nuclear membrane in a manner similar to their action on the mitochondrial outer membrane. This latter possibility is supported by our finding that Bax-induced NPR depends on the pore-forming $\alpha$-helices 5 and 6 and the oligomerization domain (required for pore formation). Targeting Bax to the mitochondria also promotes NPR, but the mechanism governing this process may differ from that of nuclear Bax. Further studies are needed to unravel the exact molecular mechanism by which Bax perforates the NE. 
The role of NPR in apoptosis is unclear. Although it has been shown in some cases to participate in the apoptotic process [5], its outcome most likely depends on cell type and the kind of apoptotic stimuli. Moreover, increased NE permeability is not enough for a nuclear matrix protein to be redistributed to the cytosol: it also needs to be released from its interaction with the nuclear components which hold it in the nucleus. Such interactions, and how apoptotic stimuli affect them, most likely depend on the cellular context and the signaling pathways induced by the apoptotic stimuli. Furthermore, although the present study focused on the redistribution of several specific nuclear proteins such as nucleolin, other proteins which might even have an anti-apoptotic effect can also be redistributed. Thus, the outcome of stress-induced NPR needs to be further studied with respect to cell type and apoptotic stimulus of interest. Nonetheless, it seems that NPR itself, at least as studied in our system here, does not induce cell death per se, since FLAG-Bax KASH was not toxic.

Our discovery of a non-canonical action of Bax on the nuclear membrane may have implications for the development of drugs based on the Bcl-2 family. So far, these drugs are $\mathrm{BH} 3$ mimetics, that is, they block the action of Bcl-2-like survival factors. Since Bcl- $\mathrm{x}_{\mathrm{L}}$ is unable to inhibit Bax-induced NPR, these compounds may not regulate this particular process. Thus, for future drug development, one needs to consider that activation or inhibition of Bax action can also affect the nucleus and that standard approaches based on the interaction between Bax and anti-apoptotic members of the Bcl-2 family may not be sufficient to obtain a therapeutically effective drug.

Acknowledgments We thank Prof. Andreas Strasser, The Walter and Eliza Hall Institute of Medical Research, Parkville, Victoria, Australia, for providing the Bax/Bak DKO MEFs, Prof. Xu Luo, Eppley Institute for Cancer Research, University of Nebraska Medical Center, Omaha, NE, USA, for providing the GFP-Bax 63-65A and GFP-Bax 92-95A plasmids, Prof. Richard J. Youle, Surgical Neurology Branch, NINDS, National Institutes of Health, Bethesda, MD, USA, for providing the Bax S184V plasmid, and Prof. Howard J. Worman, Columbia University, NY, USA, for providing the lamin A-RFP and GFP-mini-nesprin $2 \mathrm{G}$ plasmids. This work was supported by the German-Israeli Foundation (to R. Stein and C. Borner), the Spemann Graduate School of Biology and Medicine (SGBM) (GSC-4) and the Excellence Cluster BIOSS (EXC-294) funded by the DFG (to C. Borner).

Conflict of interest The authors declare that they have no conflict of interest.

\section{References}

1. Youle RJ, Strasser A (2008) The BCL-2 protein family: opposing activities that mediate cell death. Nat Rev Mol Cell Biol 9:47-59

2. Wang C, Youle RJ (2009) The role of mitochondria in apoptosis. Annu Rev Genet 43:95-118
3. Vaux DL (2011) Apoptogenic factors released from mitochondria. Biochim Biophys Acta 1813:546-550

4. Scorrano L, Korsmeyer SJ (2003) Mechanisms of cytochrome $c$ release by proapoptotic BCL-2 family members. Biochem Biophys Res Commun 304:437-444

5. Lindenboim L, Borner C, Stein R (2011) Nuclear proteins acting on mitochondria. Biochim Biophys Acta 1813:584-596

6. Lindenboim L, Blacher E, Borner C, Stein R (2010) Regulation of stress-induced nuclear protein redistribution: a new function of Bax and Bak uncoupled from Bcl-x(L). Cell Death Differ $17: 346-359$

7. Todaro GJ, Green H (1963) Quantitative studies of the growth of mouse embryo cells in culture and their development into established lines. J Cell Biol 17:299-313

8. Schinzel A, Kaufmann T, Schuler M, Martinalbo J, Grubb D, Borner C (2004) Conformational control of Bax localization and apoptotic activity by Pro168. J Cell Biol 164:1021-1032

9. Lindenboim L, Yuan J, Stein R (2000) Bcl-xS and Bax induce different apoptotic pathways in PC12 cells. Oncogene 19:1783-1793

10. Kaufmann T, Schlipf S, Sanz J, Neubert K, Stein R, Borner C (2003) Characterization of the signal that directs $\mathrm{Bcl}-\mathrm{x}(\mathrm{L})$, but not $\mathrm{Bcl}-2$, to the mitochondrial outer membrane. J Cell Biol 160:53-64

11. Lindenboim L, Borner C, Stein R (2001) Bcl-x(S) can form homodimers and heterodimers and its apoptotic activity requires localization of $\mathrm{Bcl}-\mathrm{x}(\mathrm{S})$ to the mitochondria and its $\mathrm{BH} 3$ and loop domains. Cell Death Differ 8:933-942

12. Strasser C, Grote P, Schauble K, Ganz M, Ferrando-May E (2012) Regulation of nuclear envelope permeability in cell death and survival. Nucleus 3:540-551

13. Ostlund C, Sullivan T, Stewart CL, Worman HJ (2006) Dependence of diffusional mobility of integral inner nuclear membrane proteins on A-type lamins. Biochemistry 45:1374-1382

14. Ostlund C, Folker ES, Choi JC, Gomes ER, Gundersen GG, Worman HJ (2009) Dynamics and molecular interactions of linker of nucleoskeleton and cytoskeleton (LINC) complex proteins. J cell science 122:4099-4108

15. Lindenboim L, Kringel S, Braun T, Borner C, Stein R (2005) Bak but not Bax is essential for Bcl-xS-induced apoptosis. Cell Death Differ 12:713-723

16. Phair RD, Misteli T (2000) High mobility of proteins in the mammalian cell nucleus. Nature 404:604-609

17. Boise LH, Gonzalez-Garcia M, Postema CE, Ding L, Lindsten T, Turka LA et al (1993) Bcl-x, a bcl-2-related gene that functions as a dominant regulator of apoptotic cell death. Cell 74:597-608

18. Suzuki M, Youle RJ, Tjandra N (2000) Structure of Bax: coregulation of dimer formation and intracellular localization. Cell 103:645-654

19. Zha H, Aime-Sempe C, Sato T, Reed JC (1996) Proapoptotic protein Bax heterodimerizes with Bcl-2 and homodimerizes with Bax via a novel domain (BH3) distinct from $\mathrm{BH} 1$ and $\mathrm{BH} 2$. J Biol Chem 271:7440-7444

20. Minn AJ, Kettlun CS, Liang H, Kelekar A, Vander Heiden MG, Chang BS et al (1999) Bcl-xL regulates apoptosis by heterodimerization-dependent and -independent mechanisms. EMBO J 18:632-643

21. Borner C (2003) The Bcl-2 protein family: sensors and checkpoints for life-or-death decisions. Mol Immunol 39:615-647

22. George NM, Evans JJD, Luo X (2007) A three-helix homo-oligomerization domain containing $\mathrm{BH} 3$ and $\mathrm{BH} 1$ is responsible for the apoptotic activity of Bax. Genes Dev 21:1937-1948

23. Heimlich G, McKinnon AD, Bernardo K, Brdiczka D, Reed JC, Kain R, Kronke M, Jurgensmeier JM (2004) Bax-induced cytochrome $c$ release from mitochondria depends on alpha-helices-5 and -6. Biochem J 378:247-255 
24. Upton JP, Valentijn AJ, Zhang L, Gilmore AP (2007) The N-terminal conformation of Bax regulates cell commitment to apoptosis. Cell Death Differ 14:932-942

25. Rizzuto R, Brini M, Pizzo P, Murgia M, Pozzan T (1995) Chimeric green fluorescent protein as a tool for visualizing subcellular organelles in living cells. Curr Biol CB 5:635-642

26. Nechushtan A, Smith CL, Hsu YT, Youle RJ (1999) Conformation of the Bax C-terminus regulates subcellular location and cell death. EMBO J 18:2330-2341

27. Mitoma J, Ito A (1992) The carboxy-terminal 10 amino acid residues of cytochrome b5 are necessary for its targeting to the endoplasmic reticulum. EMBO J 11:4197-4203

28. Kalderon D, Roberts BL, Richardson WD, Smith AE (1984) A short amino acid sequence able to specify nuclear location. Cell 39:499-509

29. Starr DA, Fridolfsson HN (2010) Interactions between nuclei and the cytoskeleton are mediated by SUN-KASH nuclear-envelope bridges. Annu Rev Cell Dev Biol 26:421-444

30. Goldman RD, Gruenbaum Y, Moir RD, Shumaker DK, Spann TP (2002) Nuclear lamins: building blocks of nuclear architecture. Genes Dev 16:533-547

31. Youle RJ, Karbowski M (2005) Mitochondrial fission in apoptosis. Nat Rev Mol Cell Biol 6:657-663

32. Scorrano L, Oakes SA, Opferman JT, Cheng EH, Sorcinelli MD, Pozzan T, Korsmeyer SJ (2003) BAX and BAK regulation of endoplasmic reticulum $\mathrm{Ca} 2+:$ a control point for apoptosis. Science 300: 135-139
33. Hoetelmans R, van Slooten HJ, Keijzer R, Erkeland S, van de Velde CJ, Dierendonck JH (2000) Bcl-2 and Bax proteins are present in interphase nuclei of mammalian cells. Cell Death Differ 7:384-392

34. Hoetelmans RW (2004) Nuclear partners of Bcl-2: bax and PML. DNA Cell Biol 23:351-354

35. Gajkowska B, Motyl T, Olszewska-Badarczuk H, Gniadecki R, Koronkiewicz M (2000) Structural association of Bax with nuclear matrix and cytomatrix revealed by embedment-free immunogold electron microscopy. Cell Biol Int 24:649-656

36. Gajkowska B, Motyl T, Olszewska-Badarczuk H, Godlewski MM (2001) Expression of BAX in cell nucleus after experimentally induced apoptosis revealed by immunogold and embedment-free electron microscopy. Cell Biol Int 25:725-733

37. Gajkowska B, Wojewodzka U, Gajda J (2004) Translocation of Bax and Bid to mitochondria, endoplasmic reticulum and nuclear envelope: possible control points in apoptosis. J Mol Histol 35:11-19

38. Mandal M, Adam L, Mendelsohn J, Kumar R (1998) Nuclear targeting of Bax during apoptosis in human colorectal cancer cells. Oncogene 17:999-1007

39. Wang X, Olberding KE, White C, Li C (2011) Bcl-2 proteins regulate ER membrane permeability to luminal proteins during ER stress-induced apoptosis. Cell Death Differ 18:38-47

40. Gilchrist S, Gilbert N, Perry P, Ostlund C, Worman HJ, Bickmore WA (2004) Altered protein dynamics of disease-associated lamin A mutants. BMC Cell Biol 5:46 